\title{
Exponential Stability of Stochastic Systems with Delay and Poisson Jumps
}

\author{
Wenli Zhu, Jiexiang Huang, and Zhao Zhao \\ School of Economic Mathematics, Southwestern University of Finance and Economics, Chengdu 611130, China \\ Correspondence should be addressed to Wenli Zhu; zhuwl@swufe.edu.cn
}

Received 6 April 2014; Revised 3 June 2014; Accepted 21 June 2014; Published 5 August 2014

Academic Editor: Guangchen Wang

Copyright ( 2014 Wenli Zhu et al. This is an open access article distributed under the Creative Commons Attribution License, which permits unrestricted use, distribution, and reproduction in any medium, provided the original work is properly cited.

\begin{abstract}
This paper focuses on the model of a class of nonlinear stochastic delay systems with Poisson jumps based on Lyapunov stability theory, stochastic analysis, and inequality technique. The existence and uniqueness of the adapted solution to such systems are proved by applying the fixed point theorem. By constructing a Lyapunov function and using Doob's martingale inequality and Borel-Cantelli lemma, sufficient conditions are given to establish the exponential stability in the mean square of such systems, and we prove that the exponentially stable in the mean square of such systems implies the almost surely exponentially stable. The obtained results show that if stochastic systems is exponentially stable and the time delay is sufficiently small, then the corresponding stochastic delay systems with Poisson jumps will remain exponentially stable, and time delay upper limit is solved by using the obtained results when the system is exponentially stable, and they are more easily verified and applied in practice.
\end{abstract}

\section{Introduction}

In nature, physics, society, engineering, and so on we always meet two kinds of functions with respect to time: one is deterministic and another is random. Stochastic differential equations (SDEs for short) were first initiated and developed by K. Itô [1]. Today they have become a very powerful tool applied to mathematics, physics, biology, finance, and so forth.

Currently, the study of analysis and synthesis of stochastic time delay systems, described by stochastic delayed differential equations (SDDE for short), is a popular topic in the field of control theory [2-8]. Delays in the dynamics can represent memory or inertia in the financial system [9]. Because the existence of time delay is the main reason about bringing instability and deteriorating the control performance, the study on time delay systems stability and control has important theoretical and practical values. Furthermore, it often happens in real lives that a stochastic system jumps from a "normal state" or "good state" to a "bad state," and the strength of system is random. For this class of systems, it is natural and necessary to include a jump term in them. The effect of Poisson jumps should be taken into account when studying the stability of SDEs [10-16]. Therefore, except stochastic and delay effects, Poisson jumps' effects is likely to exist widely in variety of evolution processes in which states are changed abruptly at some moments of time, including such fields as finance, economy, medicine, electronics, and so forth. Then, it is natural to consider the effect of Poisson jumps when studying the stability of SDDEs.

So far, these topics have received a lot of attention and there are so many references about them. For instance, [2-8] established some stability criteria of the stochastic systems with delay by using Lyapunov function method or Razumikhin technique or inequality technique and so on. By using the fixed point theory and Borel-Cantelli lemma, Guo and Zhu [13] studied that the solution to a class of stochastic Volterra-Levin equations with Poisson jumps is not only existent and unique but also $p$ th moment exponentially stable. By constructing a novel Lyapunov-Krasovskii functional and using some new approaches and techniques, Zhu and Cao [14] focused on the exponential stability for a class of Markovian jump impulsive stochastic Cohen-Grossberg neural networks with mixed time delays and got several novel sufficient conditions. By applying a Lyapunov-Krasovskii functional, the stochastic analysis theory, and LMI approach, Zhu and Cao [15] investigated a class of stochastic neural networks with both Markovian jump parameters and mixed 
time delays and derived some novel sufficient conditions. In [16], Zhu proposed several good sufficient conditions under which he proved the asymptotic stability in the $p$ th moment and almost sure stability of the SDEs with Lévy noise. Based on fixed point theory, Chen et al. [17] proved that the mild solution to a class of impulsive SPDEs with delays and Poisson jumps is not only existent and unique but also $p$ th moment exponentially stable.

Delay and Poisson jumps always coexist in real dynamic systems. Thus, it is reasonable to consider them together, leading us to investigate SDDEs with Poisson jumps. However, the delayed response gives us more difficulties to deal with the delayed stochastic control problems, not only for the infinite-dimensional problem, but also for the absence of Itô's formula to deal with the delayed part of the trajectory. So the stochastic controlled delay systems are more complicated. Because Lévy processes are not continuous, but their sample paths are right-continuous and have a number of random jump discontinuities occurring at random times, on each finite time interval. Since Lévy noise has more advantages than the standard Gausian noise despite its increased mathematical complexity, it is very interesting and challenging to study SDDEs with Lévy noise. There is little literature focusing on a certain class of this system, [14-17], that discussed the exponential stability of the trivial solution for this system, but these stable conditions only ensure the exponential stability of the respective solution and do not give a bound for the time delay $\delta$, and Chen et al. pointed out that it is impossible to analyze the stability of mild solutions to SDDEs by Lyapunov method.

The main objective of this paper is to fill this gap. We investigate not only the exponential stability in the mean square but also the almost surely exponential stability for a class of SDDE with Poisson jumps based on Lyapunov stability theory, Itô formula, stochastic analysis, and inequality technique. We first consider the existence and uniqueness of the adapted solution by employing fixed point theorem. Next, some sufficient conditions of exponential stability and corollaries for SDDE with Poisson jumps are obtained by using Lyapunov function. By utilizing Doob's martingale inequality and Borel-Cantelli lemma, it is shown that the exponentially stable in the mean square of SDDE with Poisson jumps implies the almost surely exponentially stable. Our results generalize and improve some recent results (for instance [5-8, 14-17]). In particular, our results show that if SDE is exponentially stable and the time delay is sufficiently small, then the corresponding SDDE with Poisson jumps will remain exponentially stable. Moreover, when the system is exponentially stable, the time delay upper limit is solved by using our results which are more easily verified and applied in practice. Our approach in the current paper is different from the above [14-17]. Finally, we present a simple example to illustrate the effectiveness of our stable results.

The rest of this paper is organized as follows. In Section 2, we give the preliminary results about SDDE with Poisson jumps. Main results and proofs for SDDE with Poisson jumps are provided in Section 3. Section 4 presents a simple example to illustrate our stable results. Section 5 lists some concluding remarks.

\section{Preliminaries}

Throughout this paper and unless specified, we let $B(t)=$ $B(t, \omega)$ be an $m$-dimensional motion and $\widetilde{N}(d t, d z)=$ $N(d t, d z)-v(d z) d t$ which is the $l$-dimensional compensated jump measure of $\eta(\cdot)$ an independent compensated Poisson random measure on a filtered probability space $\left(\Omega, \mathscr{F},\left\{\mathscr{F}_{t}\right\}_{0 \leq t \leq T}, P\right) . N(d t, d z)$ is the $l$-dimensional jump measure and $\nu(d z)$ is the Lévy measure of $l$-dimensional Lévy process $\eta(\cdot)$ and $T>0$.

We denote the notation $|\cdot|$ for the Euclidean norm. If $A$ is a vector or matrix, its transpose is denoted by $A^{T}$. If $A$ is a square matrix, the trace of $A$ is denoted by $\operatorname{tr}(A)$ and then the operator norm of $A$ is denoted by $\|A\|$; that is, $\|A\|=\sqrt{\operatorname{tr}\left(A^{T} A\right)}$. We also use the notation: $L_{\mathscr{F}}^{2}\left([s, r] ; \mathbf{R}^{n}\right)=$ $\left\{\phi(t):\{\phi(t), s \leq t \leq r\}\right.$ which is $\mathbf{R}^{n}$-valued adapted stochastic processes s.t. $\left.\int_{s}^{r} E|\phi(t)|^{2} d t<\infty\right\}$.

Suppose $X(t) \in \mathbf{R}^{n}$ is an Itô-Lévy process of the form

$$
\begin{array}{cl}
d X(t)= & b(t, X(t), Y(t), \omega) d t \\
+ & \\
+ & \\
+\int_{\mathbf{R}_{0}^{n}} \gamma(t, X(t), Y(t), \omega) d B(t) & \\
& t \in[0, T], \\
X(t)=\xi(t) ; \quad t \in[-\delta, 0], &
\end{array}
$$

where $Y(t)=X(t-\delta), \mathbf{R}_{0}^{n}:=\mathbf{R}^{n} /\{0\}$, and $\delta>0$. Here $b$ : $[0, T] \times \mathbf{R}^{n} \times \mathbf{R}^{n} \times \Omega \rightarrow \mathbf{R}^{n}, \sigma:[0, T] \times \mathbf{R}^{n} \times \mathbf{R}^{n} \times \Omega \rightarrow \mathbf{R}^{n \times m}$, and $\gamma:[0, T] \times \mathbf{R}^{n} \times \mathbf{R}^{n} \times \mathbf{R}_{0}^{n} \times \Omega \rightarrow \mathbf{R}^{n \times l}$, are given functions such that for all $t, b(t, x, y, \cdot), \sigma(t, x, y, \cdot)$, and $\gamma(t, x, y, z, \cdot)$ are $\mathscr{F}_{t}$-measurable for all $x \in \mathbf{R}^{n}, y \in \mathbf{R}^{n}$ and $z \in \mathbf{R}_{0}^{n}$. In the following, we suppress the $\omega$, for notational simplicity. The initial date $X(t)=\xi(t)$ is satisfied with $\xi:=\{\xi(s):-\delta \leq s \leq$ $0\} \in L_{\mathscr{F}_{0}}^{p}\left([-\delta, 0] ; \mathbf{R}^{n}\right)$.

Now let us present an existence and uniqueness result for (1)-(2). First we let the maps $b(t, x, y, \cdot), \sigma(t, x, y, \cdot)$, and $\gamma(t, x, y, z, \cdot)$ satisfy the following conditions.

(H2.1) At most linear growth: there exists a constant $C_{1}>0$ such that

$$
\begin{aligned}
& |b(t, x, y)|^{2}+\|\sigma(t, x, y)\|^{2} \\
& \quad+\int_{\mathbf{R}_{0}} \sum_{k=1}^{l}\left|\gamma^{(k)}\left(t, x, y, z_{k}\right)\right|^{2} \nu_{k}\left(d z_{k}\right) \\
& \quad \leq C_{1}\left(1+|x|^{2}+|y|^{2}\right)
\end{aligned}
$$

for all $x \in \mathbf{R}^{n}, y \in \mathbf{R}^{n}$, where $\gamma^{(k)} \in \mathbf{R}^{n}$ is column number $k$ of the $n \times l$ matrix $\gamma=\left[\gamma_{i k}\right]$ and $\gamma_{i}^{(k)}=\gamma_{i k}$ is the coordinate number $i$ of $\gamma^{(k)}$, and $\gamma^{(k)}(t, x, y, z)=$ $\gamma^{(k)}\left(t, x, y, z_{k}\right) ; z=\left(z_{1}, \ldots, z_{l}\right) \in \mathbf{R}^{l}$. 
(H2.2) Lipschitz continuity: there exists a constant $C_{2}>0$ such that

$$
\begin{aligned}
& \left|b(t, x, y)-b\left(t, x^{\prime}, y^{\prime}\right)\right|^{2}+\left\|\sigma(t, x, y)-\sigma\left(t, x^{\prime}, y^{\prime}\right)\right\|^{2} \\
& \quad+\int_{\mathbf{R}_{0}} \sum_{k=1}^{l}\left|\gamma^{(k)}\left(t, x, y, z_{k}\right)-\gamma^{(k)}\left(t, x^{\prime}, y^{\prime}, z_{k}\right)\right|^{2} \nu_{k}\left(d z_{k}\right) \\
& \quad \leq C_{2}\left(\left|x-x^{\prime}\right|^{2}+\left|y-y^{\prime}\right|^{2}\right)
\end{aligned}
$$

for all $x \in \mathbf{R}^{n}, y \in \mathbf{R}^{n}$.

Lemma 1 (see [18]). For any real matrices $\zeta_{1}, \zeta_{2} \in R^{n}$ and a constant $\theta>0$, the following matrix inequality holds:

$$
2 \zeta_{1}^{T} \zeta_{2} \leq \theta \zeta_{1}^{T} \zeta_{1}+\frac{1}{\theta} \zeta_{2}^{T} \zeta_{2}
$$

Theorem 2. Let (H2.1) and (H2.2) hold. Then for any $\xi(t) \epsilon$ $L_{\mathscr{F}_{0}}^{2}\left([-\delta, 0] ; \mathbf{R}^{n}\right)$, (1)-(2) have a unique adapted solution $X(t ; \xi)$ such that

$$
E\left[|X(t ; \xi)|^{2}\right]<\infty
$$

for all $t$. When $b(t, 0,0,0)=\sigma(t, 0,0,0) \equiv 0$, it is easy to see that (1)-(2) have a trivial solution $X(t ; 0)=0$.

We present the proof of Theorem 2 which is left in Appendix.

To develop our theories and results, we need to introduce the following concepts. For stochastic system, exponential stability in mean square and almost surely exponential stability are generally used [7].

Definition 3. The trivial solution of (1)-(2) is said to be $p$ th moment exponentially stable. If there exists a positive constant $\varepsilon$ such that

$$
\limsup _{t \rightarrow \infty} \frac{1}{t} \ln \left(E|X(t ; \xi)|^{p}\right) \leq-\varepsilon
$$

for any $\xi \in L_{\mathscr{F}_{0}}^{p}\left([-\delta, 0] ; \mathbf{R}^{n}\right)$.

Particularly, $p=2$; it is called mean square exponentially stable.

Definition 4. The trivial solution of (1)-(2) is said to be almost surely exponentially stable. If there exists a positive constant $\eta$ such that

$$
\limsup _{t \rightarrow \infty} \frac{1}{t} \ln |X(t ; \xi)| \leq-\eta \quad \text { a.s. }
$$

for any $\xi \in L_{\mathscr{F}_{0}}^{p}\left([-\delta, 0] ; \mathbf{R}^{n}\right)$.

\section{Main Results}

For simplicity, in what follows we write $X(t ; \xi)=X(t)$.

We make the following assumptions for the coefficients of (1)-(2).
In the study of mean square exponential stability, it is often to use a quadratic function as the Lyapunov function; that is, $V(t, x)=x^{T} G x$, where $G$ is a symmetric positive definite $n \times n$ matrix.

Theorem 5. Let (H2.1)-(H2.2) hold; then the trivial solution of (1)-(2) is exponentially stable in the mean square. Assume that there exists a symmetric positive definite $n \times n$ matrices $G$ and a constant $\lambda>0$ such that

$$
2 X^{T} G b(t, X, X) \leq-\lambda|X|^{2}, \quad \forall(t, x, y) \in[0, T] \times R^{n} \times R^{n},
$$

$$
\lambda>4\|G\| C_{1}+2\|G\| \sqrt{6 C_{1} C_{2} \delta(\delta+2)} .
$$

In order to prove Theorem 5, we need two lemmas, proofs of which are left in Appendix.

Lemma 6. Fix the initial data $\xi(t)$ arbitrarily. Then,

$$
\begin{aligned}
& \int_{\delta}^{t} e^{\varepsilon s} \int_{s-\delta}^{s} E|X(\tau)|^{2} d \tau d s \leq \delta e^{\varepsilon \delta} \int_{0}^{t} e^{\varepsilon \tau} E|X(\tau)|^{2} d \tau \\
& \int_{0}^{t} e^{\varepsilon s} E|X(s-\delta)|^{2} d s \leq c_{11} e^{\varepsilon \delta}+e^{\varepsilon \delta} \int_{0}^{t} e^{\varepsilon s} E|X(s)|^{2} d s
\end{aligned}
$$

for any $t \geq \delta$, where $c_{11}$ is a constant larger than $\int_{-\delta}^{0} E|\xi(s)|^{2} d s$.

Lemma 7. Let (H2.1) and (H2.2) hold. Fix the initial data $\xi(t)$ arbitrarily; then,

$$
\begin{aligned}
& \int_{\delta}^{t} e^{\varepsilon s} \int_{s-\delta}^{s} E|X(\tau-\delta)|^{2} d \tau d s \\
& \leq \delta c_{11} e^{2 \varepsilon \delta}+\delta e^{2 \varepsilon \delta} \int_{0}^{t} e^{\varepsilon s} E|X(s)|^{2} d s \\
& \int_{0}^{t} e^{\varepsilon s} E|X(s)-X(s-\delta)|^{2} d s \leq c_{22} \\
& \quad+3 C_{1}(\delta+2) \delta e^{\varepsilon \delta} \int_{0}^{t} e^{\varepsilon s} E|X(s)|^{2} d s \\
& \quad+3 C_{1}(\delta+2)\left(c_{11} \delta e^{2 \varepsilon \delta}+\delta e^{2 \varepsilon \delta} \int_{0}^{t} e^{\varepsilon s} E|X(s)|^{2} d s\right)
\end{aligned}
$$

for any $t \geq \delta$, where

$$
\begin{aligned}
c_{22} \geq & \int_{0}^{\delta} e^{\varepsilon s} E|X(s)-X(s-\delta)|^{2} d s \\
& +\frac{3 \delta C_{1}(\delta+2)}{\varepsilon}\left(e^{\varepsilon T}-e^{\varepsilon \delta}\right) .
\end{aligned}
$$

Based on Lemmas 6 and 7 above, we now carry out a proof for Theorem 5 . 
Proof of Theorem 5. Fix the initial data $\xi(t)$ arbitrarily. Applying Itô's formula to $X^{T} G X$, we have

$$
\begin{aligned}
d\left(X^{T} G X\right)= & 2 X^{T} G b(t, X, Y) d t \\
& +2 X^{T} G \sigma(t, X, Y) d B(t) \\
& +\operatorname{tr}\left[\sigma^{T}(t, X, Y) G \sigma(t, X, Y)\right] d t \\
& +\sum_{k=1}^{l} \int_{\left|z_{k}\right|<R} \gamma^{(k)}\left(t, X, Y, z_{k}\right) \\
& \times G \gamma^{(k)}\left(t, X, Y, z_{k}\right) v_{k}\left(d z_{k}\right) d t \\
& +\sum_{k=1}^{l} \int_{\mathbf{R}_{0}}\left\{\gamma^{(k)}\left(t, X, Y, z_{k}\right) G \gamma^{(k)}\left(t, X, Y, z_{k}\right)\right. \\
& +X^{T}\left(t^{-}\right) G \gamma^{(k)}\left(t, X, Y, z_{k}\right) \\
& \left.+\gamma^{(k)}\left(t, X, Y, z_{k}\right) G X\left(t^{-}\right)\right\} \\
& \times \widetilde{N}_{k}\left(d t, d z_{k}\right) .
\end{aligned}
$$

Applying Itô's formula to $e^{\varepsilon t} X^{T} G X$ and taking the expectation, we have

$$
\begin{aligned}
E\left(e^{\varepsilon t} X^{T} G X\right) \leq & E\left(\xi^{T}(0) G \xi(0)\right) \\
& +\varepsilon E \int_{0}^{t} e^{\varepsilon s} X^{T} G X d s \\
& +E \int_{0}^{t} e^{\varepsilon s} 2 X^{T} G b(s, X, Y) d s \\
& +E \int_{0}^{t} e^{\varepsilon s} \operatorname{tr}\left[\sigma^{T}(s, X, Y) G \sigma(s, X, Y)\right] d s \\
& +E \int_{0}^{t} e^{\varepsilon s}\left[\sum_{k=1}^{l} \int_{\left|z_{k}\right|<R} \gamma^{(k)}\left(s, X, Y, z_{k}\right)\right. \\
& \times G \gamma^{(k)}\left(s, X, Y, z_{k}\right) \\
& +\varepsilon E \int_{0}^{t} e^{\varepsilon s} X^{T} G X d s+I_{1}+I_{2}+I_{3}, \\
& E\left(\xi_{k}^{T}\left(d z_{k}\right)\right] d s
\end{aligned}
$$

where

$$
\begin{aligned}
& I_{1}=E \int_{0}^{t} e^{\varepsilon s} 2 X^{T} G b(s, X, Y) d s, \\
& I_{2}=E \int_{0}^{t} e^{\varepsilon s} \operatorname{tr}\left[\sigma^{T}(s, X, Y) G \sigma(s, X, Y)\right] d s,
\end{aligned}
$$

$$
\begin{aligned}
I_{3}=E \int_{0}^{t} e^{\varepsilon s}\left[\sum_{k=1}^{l} \int_{\left|z_{k}\right|<R}\right. & \gamma^{(k)}\left(t, X, Y, z_{k}\right) \\
& \left.\times G \gamma^{(k)}\left(t, X, Y, z_{k}\right) v_{k}\left(d z_{k}\right)\right] d s .
\end{aligned}
$$

Combining Lemma 1 and (9) as well as (H2.2), we can estimate $I_{1}$ as follows:

$$
\begin{aligned}
I_{1}=E \int_{0}^{t} e^{\varepsilon s}\{ & 2 X^{T} G b(s, X, X) \\
& \left.+2 X^{T} G[b(s, X, Y)-b(s, X, X)]\right\} d s \\
\leq E \int_{0}^{t} e^{\varepsilon s}\left\{-\lambda|X|^{2}+\theta|X|^{2}\right. & \\
& \left.+\left(\frac{\|G\|^{2}}{\theta}\right) \cdot C_{2}|X-Y|^{2}\right\} d s .
\end{aligned}
$$

where $\theta>0$ is a constant.

By (H2.1), $I_{2}+I_{3}$ of (17) yields

$$
\begin{aligned}
I_{2}+I_{3} \leq & 2 C_{1}\|G\|\left(e^{\varepsilon T}-1\right) \\
& +2 C_{1}\|G\| \int_{0}^{t} e^{\varepsilon s}\left(E|X|^{2}+E|Y|^{2}\right) d s .
\end{aligned}
$$

Substituting the above two into (17) and using Lemmas 6 and 7, we get an estimate of $E\left(e^{\varepsilon t} X^{T} G X\right)$ as follows:

$$
\begin{aligned}
& E\left(e^{\varepsilon t} X^{T} G X\right) \\
& \leq E\left(\xi^{T}(0) G \xi(0)\right)+2 C_{1}\|G\| e^{\varepsilon T} \\
& \quad-\left[\lambda-\theta-\|G\|\left(2 C_{1}+\varepsilon\right)\right] \int_{0}^{t} e^{\varepsilon s} E|X|^{2} d s \\
& +2 C_{1}\|G\|\left(c_{11} e^{\varepsilon \delta}+e^{\varepsilon \delta} \int_{0}^{t} e^{\varepsilon s} E|X|^{2} d s\right) \\
& +\left(\frac{\|G\|^{2}}{\theta}\right) \cdot C_{2}\left[c_{22}+3 C_{1}(\delta+2) \delta e^{\varepsilon \delta}\right. \\
& \quad \times \int_{0}^{t} e^{\varepsilon s} E|X|^{2} d s+3 C_{1}(\delta+2) \\
& \left.\times\left(c_{11} \delta e^{2 \varepsilon \delta}+\delta e^{2 \varepsilon \delta} \int_{0}^{t} e^{\varepsilon s} E|X|^{2} d s\right)\right] \\
& =c_{33} .
\end{aligned}
$$

for $t \geq \delta$, where

$$
\begin{aligned}
c_{33}= & E\left(\xi^{T}(0) G \xi(0)\right)+2 C_{1}\|G\| e^{\varepsilon T}+2 C_{1} c_{11}\|G\| e^{\varepsilon \delta} \\
& +\frac{\left(\|G\| \sqrt{C_{2}}\right)^{2}}{\theta} \cdot\left[c_{22}+3 C_{1} c_{11} \delta(\delta+2) e^{2 \varepsilon \delta}\right) .
\end{aligned}
$$


For small enough $\varepsilon>0$, we derive

$$
\begin{aligned}
& \|G\|\left(2 C_{1}+\varepsilon\right)+\theta+2 C_{1}\|G\| e^{\varepsilon \delta} \\
& +3 \frac{\left(\|G\| \sqrt{C_{1} C_{2}(\delta+2) \delta}\right)^{2}}{\theta} \cdot\left(e^{\varepsilon \delta}+e^{2 \varepsilon \delta}\right) \\
& \geq 4\|G\| C_{1}+\theta \\
& +\frac{\left(\|G\| \sqrt{6 C_{1} C_{2} \delta(\delta+2)}\right)^{2}}{\theta} .
\end{aligned}
$$

If (10) holds, then we can choose $\varepsilon>0$ small enough such that

$$
\begin{aligned}
\lambda= & \|G\|\left(2 C_{1}+\varepsilon\right)+\theta+2 C_{1}\|G\| e^{\varepsilon \delta} \\
& +3 \frac{\left(\|G\| \sqrt{C_{1} C_{2}(\delta+2) \delta}\right)^{2}}{\theta} \cdot\left(e^{\varepsilon \delta}+e^{2 \varepsilon \delta}\right) .
\end{aligned}
$$

Since $G$ is positive definite,

$$
X^{T} G X \geq \lambda_{\min }(G)|X|^{2},
$$

where $\lambda_{\min }(G)>0$ is the smallest eigenvalue of $G$.

Then,

$$
E\left(e^{\varepsilon t} X^{T} G X\right) \geq E\left(e^{\varepsilon t} \lambda_{\min }(G)|X|^{2}\right) .
$$

It then follows from (21) that

$$
\begin{aligned}
\frac{1}{t} \ln \left(E|X(t)|^{2}\right) & \leq \frac{1}{t} \ln \left(\left[\frac{c_{44}}{\lambda_{\min }(G)}\right] e^{-\varepsilon t}\right) \\
& =-\varepsilon+\frac{1}{t} \ln \left[\frac{c_{44}}{\lambda_{\min }(G)}\right] .
\end{aligned}
$$

This easily yields

$$
\limsup _{t \rightarrow \infty} \frac{1}{t} \ln \left(E|X(t)|^{2}\right) \leq-\varepsilon
$$

Then (1)-(2) is exponentially stable in the mean square.

Theorem 8. Let $\varepsilon>0$, under the same assumption as Theorem 5. If inequality (28) holds, then,

$$
\limsup _{t \rightarrow \infty} \frac{1}{t} \ln (|X(t)|) \leq-\frac{\varepsilon}{2} \quad \text { a.s. }
$$

Proof. Let $\varepsilon>0$, under the same assumption as Theorem 5. It follows from (27) that

$$
\frac{1}{t} \ln \left(E|X(t)|^{2}\right) \leq-\varepsilon+\frac{1}{t} \cdot \ln M
$$

for all $t \geq \delta$. Here $M=c_{44} / \lambda_{\min }(G)$. Then, for $t \in[k \delta,(k+$ 1) $\delta$ ], $k=2,3, \ldots$, we have

$$
E\left(\sup _{k \delta \leq t \leq(k+1) \delta} E|X(t)|^{2}\right) \leq M e^{-\varepsilon k \delta} .
$$

Let $\varepsilon_{0} \in(0, \varepsilon)$ be arbitrary. By Doob's martingale inequality. It follows from (31) that

$$
P\left(\omega: \sup _{k \delta \leq t \leq(k+1) \delta}|X(t)|>e^{-\left(\varepsilon-\varepsilon_{0}\right) k \delta / 2}\right) \leq c_{33} e^{-\varepsilon_{0} k \delta} .
$$

Thus, it follows from the Borel-Cantelli lemma that, for almost all $\omega \in \Omega$, there exists $k_{0}(\omega)$, and $k \geq k_{0}(\omega)$,

$$
P\left(\omega: \sup _{k \delta \leq t \leq(k+1) \delta}|X(t)| \leq e^{-\left(\varepsilon-\varepsilon_{0}\right) k \delta / 2}\right)=1 .
$$

Since $\varepsilon_{0}$ is arbitrary, we must have

$$
\limsup _{t \rightarrow \infty} \frac{1}{t} \ln |X(t)| \leq-\frac{\varepsilon}{2} \quad \text { a.s. }
$$

Remark 9. The exponentially stable in the mean square of (1)(2) implies the almost surely exponentially stable. In general, Theorem 8 is still true for $p$ th moment exponential stable.

Let us single out three important special cases.

Case 1. If $\sigma=0$ and $N=0$ (no jumps), then (1)-(2) reduces to ODE with delay

$$
\begin{aligned}
d X(t) & =b(t, X, Y) d t, \quad t \in[0, T], \\
X(t) & =\xi(t), \quad t \in[-\delta, 0] .
\end{aligned}
$$

Applying Theorem 5 to (35), we obtain the following useful result.

Corollary 10. Let (H2.1)-(H2.2) hold; then the trivial solution of (35) is exponentially stable in the mean square. Assume that there exists a symmetric positive definite $n \times n$ matrices $G$ and a constant $\lambda>0$ such that

$$
2 X^{T} G b(t, X, X) \leq-\lambda|X|^{2}, \quad \forall(t, x, x) \in[0, T] \times R^{n} \times R^{n},
$$

$$
\lambda>2\|G\| \delta \sqrt{2 C_{1} C_{2}} .
$$

Case 2. If $N=0$ (no jumps), then (1)-(2) reduces to SDE with delay

$$
\begin{gathered}
d X(t)=b(t, X, Y) d t+\sigma(t, X, Y) d B(t), \quad t \in[0, T], \\
X(t)=\xi(t), \quad t \in[-\delta, 0] .
\end{gathered}
$$

Applying Theorem 5 to (38), we obtain the following useful result.

Corollary 11. Let (H2.1)-(H2.2) hold; then the trivial solution of (38) is exponentially stable in the mean square. Assume that there exists symmetric positive definite $n \times n$ matrices $G$ and $a$ constant $\lambda>0$ such that

$$
2 X^{T} G b(t, X, X) \leq-\lambda|X|^{2}, \quad \forall(t, x, x) \in[0, T] \times R^{n} \times R^{n},
$$

$$
\lambda>2\|G\| C_{1}+4\|G\| \sqrt{C_{1} C_{2} \delta(\delta+1)} .
$$


Remark 12. The bound for the time delay $\delta$ when (1)-(2) is exponentially stable which follows from (10), the bound for the corresponding deterministic case follows from (37), and the bound for the corresponding stochastic case follows from (40).

Case 3. If the time delay $\delta=0$, then (1)-(2) reduces to the nondelay SDE with jumps

$$
\begin{aligned}
d X(t)= & b(t, X, X) d t+\sigma(t, X, X) d B(t) \\
& +\int_{\mathbf{R}_{0}^{n}} \gamma(t, X, X, z, \omega) \widetilde{N}(d t, d z) ; \quad t \in[0, T] .
\end{aligned}
$$

One of the powerful techniques employed in the study of the stability problem is the method of the Lyapunov functions or functional [19]. However, it is generally much more difficult to construct the Lyapunov functionals in the case of delay than the Lyapunov functions in the case of nondelay. Therefore another useful technique has been developed, that is, to compare the stochastic differential delay equations with the corresponding nondelay equations. To explain, let us look at a SDE (1) with delay and jumps?

$$
\begin{array}{r}
d X(t)=b(t, X, Y) d t+\sigma(t, X, Y) d B(t) \\
+\int_{\mathbf{R}_{0}^{n}} \gamma\left(t, X\left(t^{-}\right), Y\left(t^{-}\right), z\right) \widetilde{N}(d t, d z) ; \\
\quad t \in[0, T] .
\end{array}
$$

Equation (1) can be rewritten as

$$
\begin{aligned}
& d X(t)=b(t, X, X) d t+\sigma(t, X, X) d B(t) \\
&+\int_{\mathbf{R}_{0}^{n}} \gamma\left(t, X\left(t^{-}\right), X\left(t^{-}\right), z\right) \widetilde{N}(d t, d z) \\
& \quad-[b(t, X, X)-b(t, X, Y)] d t \\
& \quad-[\sigma(t, X, X)-\sigma(t, X, Y)] d B(t) \\
&-\int_{\mathbf{R}_{0}^{n}}\left[\gamma\left(t, X\left(t^{-}\right), X\left(t^{-}\right), z\right)\right. \\
&\left.\quad-\gamma\left(t, X\left(t^{-}\right), Y\left(t^{-}\right), z\right)\right] \widetilde{N}(d t, d z),
\end{aligned}
$$

and regard it as the perturbed system of the corresponding nondelay SDE (41). Obviously, if the time delay $\delta$ is sufficiently small then the perturbation term,

$$
\begin{aligned}
& {[b(t, X, X)-b(t, X, Y)] d t} \\
& \quad+[\sigma(t, X, X)-\sigma(t, X, Y)] d B(t) \\
& +\int_{\mathbf{R}_{0}^{n}}\left[\gamma\left(t, X\left(t^{-}\right), X\left(t^{-}\right), z\right)\right. \\
& \left.\quad-\gamma\left(t, X\left(t^{-}\right), Y\left(t^{-}\right), z\right)\right] \widetilde{N}(d t, d z),
\end{aligned}
$$

could be so small that the perturbed equation (1) would behave in a similar way as (41) asymptotically. Applying
Theorem 5 and Remark 12 in [20], we derive (1) which will remain exponentially stable.

Corollary 13. If the nondelay equation (41) is exponentially stable and the time delay $\delta$ is sufficiently small, then the corresponding delay equation (1) will remain exponentially stable.

\section{Example}

Let us now present a simple example to illustrate our results, which can help us find the time delay upper limit.

Example 1. For simplicity of presentation, let us consider a simple one-dimensional (i.e., $n=m=l=1$, thus, the indices $i$ and $j$ in Theorem 5 will be omitted below) delay equation with jumps

$$
\begin{array}{r}
d X(t)=b(t, X, Y) d t+\sigma(t, X, Y) d B(t) \\
+\int_{\mathbf{R}_{0}} \gamma\left(t, X\left(t^{-}\right), Y\left(t^{-}\right), z\right) \widetilde{N}(d t, d z) ; \\
\quad t \in[0, T],
\end{array}
$$

where $B(t)$ is one-dimensional Brownian motion. Constants $T>0$ and $\delta>0$ is a given finite time delay. For convenience, let us choose $G=1$ in this one-dimensional case. Hence (9) is satisfied with $\lambda \geq 2$.

Moreover, we let $b(t, x, y)=-y, \sigma(t, x, y)=0.5 x-0.5 y$ and $\gamma(t, x, y, z)=z, N(d t, d z)=d N(t), \nu(d z)=\kappa f(z) d z$, where $d N(t)$ is a Poisson process with jump intensity $\kappa, f(z)$ is log-normal density: $f(z)=(1 / \sqrt{2 \pi} \omega z) e^{-(\ln z-\mu)^{2} / 2 \omega^{2}}$ with $E[z]=e^{\mu+\omega^{2} / 2}$ and $D[z]=\left(e^{\omega^{2}}-1\right) e^{2 \mu+\omega^{2}}, \mu$, is mean of jump $z$ and $\omega$ is the variance of jump $z$ and $x \in R, y \in R$. Then $\widetilde{N}(d t, d z)=d N(t)-\kappa f(z) d z d t$. Here we let $\mu=-0.9, \omega=$ 0.45 , and $\kappa=0.1$. Then

$$
\int_{\mathbf{R}}|z|^{2} \nu(d z)=\kappa E\left[z^{2}\right]=\kappa e^{2 \mu+2 \omega^{2}}=0.025 .
$$

One can write (45) as the following stochastic differential delay equation with jumps:

$$
\begin{aligned}
d X(t)= & -X(t-\delta) d t \\
& +[0.5 X(t)-0.5 X(t-\delta)] d B(t) \\
& +\int_{\mathbf{R}_{0}} z \widetilde{N}(d t, d z) .
\end{aligned}
$$

It is easy to see that hypotheses (H2.1)-(H2.1) are satisfied with $C_{1}=1.5, C_{2}=1.5$. On the other hand, it is easy to see that condition (9) is satisfied with $\lambda=8$ and (10) becomes $\delta<$ 0.037 .

Therefore, by Theorems 5 and 8, we can conclude that (47) is both mean square and almost surely exponentially stable provided $\delta<0.037$.

Particularly, $\kappa=0$; then (45) reduces to SDE with delay

$$
d X(t)=-X(t-\delta) d t+[0.5 X(t)-0.5 X(t-\delta)] d B(t) .
$$


It is easy to see that hypotheses (H2.1)-(H2.2) are satisfied with $C_{1}=1.5$ and $C_{2}=1.5$. On the other hand, it is easy to see that condition (9) is satisfied with $\lambda=8$ and (40) becomes $\delta<0.395$.

Therefore, by Corollary 11 and Theorem 8 , we can conclude that (48) is both mean square and almost surely exponentially stable provided $\delta<0.395$.

Moreover, setting $b(t, x, y)=-y, \sigma(t, x, y)=0$, and $\kappa=$ 0 , then (45) becomes

$$
d X(t)=-X(t-\delta) d t
$$

It is easy to see that hypotheses (H2.1)-(H2.2) are satisfied with $C_{1}=1$ and $C_{2}=1$. On the other hand, it is easy to see that condition (9) is satisfied with $\lambda=8$ and (37) becomes $\delta<2 \sqrt{2}$.

Therefore, by Corollary 10 and Theorem 8, we can conclude that (49) is both mean square and almost surely exponentially stable provided $\delta<2 \sqrt{2}$.

Remark 14. Figure 1 gives the simulation results of Example 1 when $\sigma \neq 0, \kappa \neq 0$, and $\delta<0.037$. The parameter values used in the calculations are $\|G\|=1, \lambda=8, C_{1}=1.5, C_{2}=1.5$, and $\delta=0.03$. Figure 2 gives the simulation results of Example 1 when $\sigma \neq 0, \kappa=0$, and $\delta<0.395$. The parameter values used in the calculations are $\|G\|=1, \lambda=8, C_{1}=1.5, C_{2}=1.5$, and $\delta=0.3$. Figure 3 gives the simulation results of Example 1 when $\sigma=0, \kappa=0$, and $\delta<2 \sqrt{2}$. The parameter values used in the calculations are $\|G\|=1, \lambda=8, C_{1}=1, C_{2}=1$, and $\delta=1$.

\section{Concluding Remarks}

In this paper, we investigate not only the exponential stability in the mean square but also the almost surely exponential stability for a class of SDDE with Poisson jumps based on Lyapunov stability theory, Itô formula, stochastic analysis, and inequality technique. We first consider the existence and uniqueness of the adapted solution by employing fixed point theorem. Next, some sufficient conditions of exponential stability and corollaries for SDDE with Poisson jumps are obtained by using Lyapunov function. By utilizing Doob's martingale inequality and Borel-Cantelli lemma, we find that the exponentially stable in the mean square of SDDE with Poisson jumps implies the almost surely exponentially stable. Our results generalize and improve some recent results ([5-8, 14-17]). In particular, our results show that if SDE is exponentially stable and the time delay is sufficiently small, then the corresponding SDDE with Poisson jumps will remain exponentially stable. Moreover, when the system is exponentially stable, the time delay upper limit is solved by using our results which are more easily verified and applied in practice. Our approach in the current paper is different from the above [14-17]. Finally, we present a simple example to illustrate the effectiveness of our stable results. Another challenging problem is to study a class of SDEs with variable delays and Poisson jumps. We hope to study these problems in forthcoming papers.

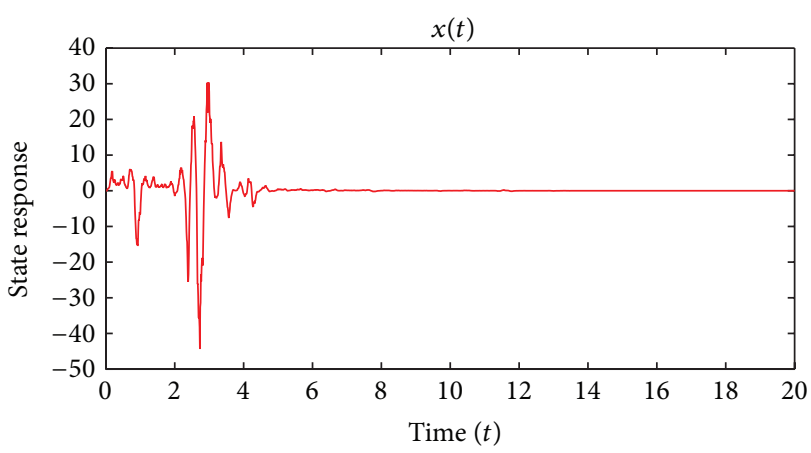

Figure 1: The simulation results of Example 1 when $\sigma \neq 0, \kappa \neq 0$, and $\delta=0.03$.

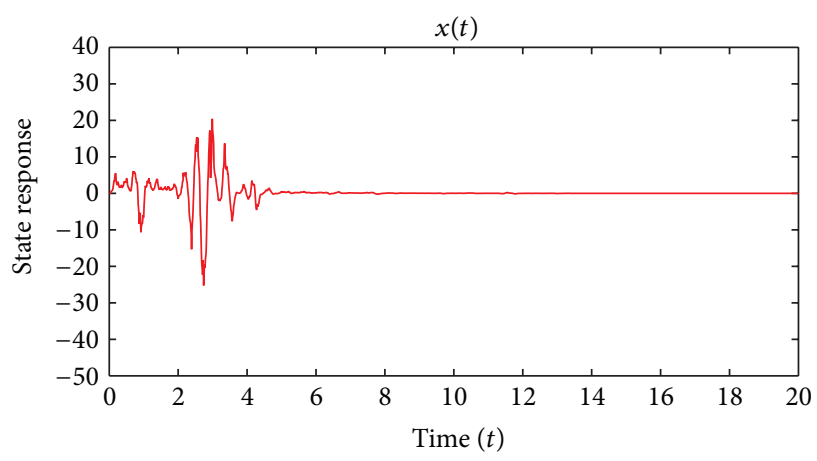

FIgURE 2: The simulation results of Example 1 when $\sigma \neq 0, \kappa=0$, and $\delta=0.3$.

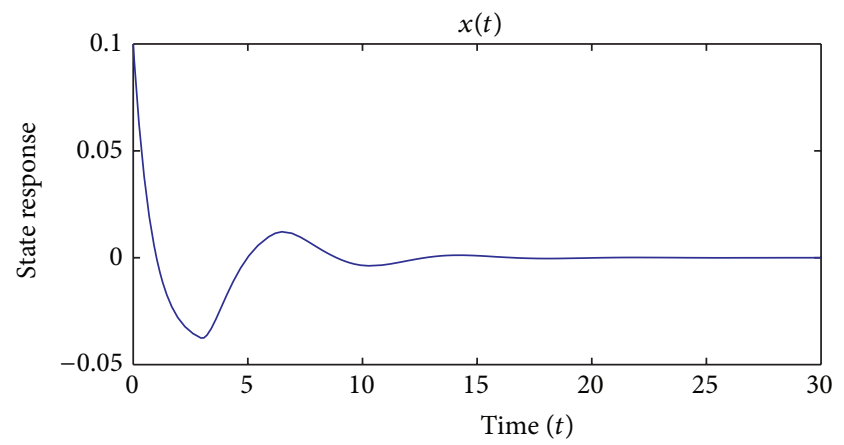

FIGURE 3: The simulation results of Example 1 when $\sigma=0, N=0$, $\delta=1$.

\section{Appendix}

We now present proof of Theorem 2 .

Proof of Theorem 2. Let us define a norm in Banach space $L_{\mathscr{F}}^{2}\left([-\delta, T] ; \mathbf{R}^{n}\right)$ as follows:

$$
|\chi(\cdot)|_{\vartheta}=\left(E\left[\int_{-\delta}^{T} e^{-\vartheta s}|\chi(s)|^{2} d s\right]\right)^{1 / 2}, \quad \vartheta>0
$$


Clearly it is equivalent to the original norm of $L_{\mathscr{F}}^{2}\left([-\delta, T] ; \mathbf{R}^{n}\right)$. We consider

$$
\begin{aligned}
& X(t)= \xi(0)+\int_{0}^{t} b\left(s, \chi, y_{\chi}\right) d s \\
&+\int_{0}^{t} \sigma\left(s, \chi, y_{\chi}\right) d B(s) \\
&+\int_{\mathbf{R}_{0}^{n}} \gamma\left(s, X\left(s^{-}\right), Y\left(s^{-}\right), z\right) \widetilde{N}(d s, d z) ; \\
&X(t)=\xi(t), \quad t \in[-\delta, 0], T]
\end{aligned}
$$

where $y_{\chi}=\chi(t-\delta)$. Define a mapping $\mathbb{\mathbb { T }}: L_{\mathscr{F}}^{2}\left([-\delta, T] ; \mathbf{R}^{n}\right) \rightarrow$ $L_{\mathscr{F}}^{2}\left([-\delta, T] ; \mathbf{R}^{n}\right)$ such that $\mathbb{T}(\chi(\cdot))=X(\cdot)$. We desire to prove that $\mathbb{T}$ is a contraction mapping under the norm $|\chi(\cdot)|_{9}$. For arbitrary $\chi(\cdot), \chi^{\prime}(\cdot) \in L_{\mathscr{F}}^{2}\left([-\delta, T] ; \mathbf{R}^{n}\right)$, set $\mathbb{T}(\chi(\cdot))=$ $X(\cdot), \mathbb{T}\left(\chi^{\prime}(\cdot)\right)=\chi^{\prime}(\cdot)$, and $\widehat{\chi}^{(\cdot)}=\chi(\cdot)-\chi^{\prime}(\cdot), \widehat{X}(\cdot)=X(\cdot)-$ $X^{\prime}(\cdot)$. Then, $\widehat{X}(\cdot)$ satisfies

$$
\begin{aligned}
& \widehat{X}(\cdot)=\int_{0}^{t}\left[b\left(s, \chi, y_{\chi}\right)-b\left(s, \chi^{\prime}, y_{\chi}^{\prime}\right)\right] d s \\
&+\quad \int_{0}^{t}\left[\sigma\left(s, \chi, y_{\chi}\right)-\sigma\left(s, \chi^{\prime}, y_{\chi}^{\prime}\right)\right] d B(s)+\int_{\mathbf{R}_{0}^{n}}\left[\gamma\left(s, \chi\left(s^{-}\right), y_{\chi}\left(s^{-}\right), z\right)\right. \\
&\left.-\gamma\left(s, \chi^{\prime}\left(s^{-}\right), y_{\chi}^{\prime}\left(s^{-}\right), z\right)\right] \widetilde{N}(d s, d z) ; \\
& t \geq 0,
\end{aligned}
$$$$
\widehat{X}(\cdot)=0, \quad t \in[-\delta, 0] .
$$

Applying Itô's formula to $e^{-9 t}|\widehat{X}(t)|^{2}$ and taking the expectation, we have

$$
\begin{aligned}
& \vartheta E \int_{0}^{T} e^{-\vartheta t}|\widehat{X}(t)|^{2} d t \\
& =2 E \int_{0}^{T} e^{-\vartheta t} \widehat{X}(t)\left|b\left(t, \chi, y_{\chi}\right)-b\left(t, \chi^{\prime}, y_{\chi}^{\prime}\right)\right| d t \\
& \quad+E \int_{0}^{T} e^{-\vartheta t}\left|\sigma\left(s, \chi, y_{\chi}\right)-\sigma\left(s, \chi^{\prime}, y_{\chi}^{\prime}\right)\right|^{2} d t \\
& \quad+E \int_{0}^{T} \sum_{k=1}^{l} \int_{\left|z_{k}\right|<R} e^{-\vartheta t} \mid \gamma^{(k)}\left(t, \chi\left(t^{-}\right), y_{\chi}\left(t^{-}\right), z_{k}\right) \\
& \quad-\left.\gamma^{(k)}\left(t, \chi^{\prime}\left(t^{-}\right), y_{\chi}^{\prime}\left(t^{-}\right), z_{k}\right)\right|^{2}
\end{aligned}
$$$$
\times v_{k}\left(d z_{k}\right) d t .
$$

Lemma 1 yields

$$
\begin{aligned}
& \vartheta E \int_{0}^{T} e^{-\vartheta t}|\widehat{X}(t)|^{2} d t \\
& \leq E \int_{0}^{T} e^{-\vartheta t}\left(|\widehat{X}(t)|^{2}+\left|b\left(t, \chi, y_{\chi}\right)-b\left(t, \chi^{\prime}, y_{\chi}^{\prime}\right)\right|^{2}\right) d t \\
& \quad+E \int_{0}^{T} e^{-\vartheta t}\left|\sigma\left(s, \chi, y_{\chi}\right)-\sigma\left(s, \chi^{\prime}, y_{\chi}^{\prime}\right)\right|^{2} d t \\
& \quad+E \int_{0}^{T} \sum_{k=1}^{l} \int_{\left|z_{k}\right|<R} e^{-9 t} \mid \gamma^{(k)}\left(t, \chi\left(t^{-}\right), y_{\chi}\left(t^{-}\right), z_{k}\right) \\
& \quad \times v_{k}\left(d z_{k}\right) d t .
\end{aligned}
$$

Then by (H2.2), we obtain

$$
\begin{aligned}
(\vartheta-1) E \int_{0}^{T} e^{-\vartheta t}|\widehat{X}(t)|^{2} d t & \\
\leq 3 C_{2} \cdot E & {\left[\int_{0}^{T} e^{-\vartheta t}|\widehat{\chi}(t)|^{2} d t\right.} \\
& \left.+\int_{0}^{T} e^{-\vartheta t}\left|\widehat{y}_{\chi}(t)\right|^{2} d t\right],
\end{aligned}
$$

where

$$
\begin{aligned}
& \int_{0}^{T} e^{-\vartheta t}\left|\widehat{y}_{\chi}(t)\right|^{2} d t \underline{\underline{\underline{\tau=t-\delta}}} \\
& \quad=e^{-\theta \delta} \int_{-\delta}^{T-\delta} e^{-\theta \tau}|\widehat{\chi}(\tau)|^{2} d \tau \leq \int_{-\delta}^{T-\delta} e^{-\vartheta \tau}|\widehat{\chi}(\tau)|^{2} d \tau \\
& \quad \leq \int_{-\delta}^{T} e^{-\vartheta \tau}|\widehat{\chi}(\tau)|^{2} d \tau .
\end{aligned}
$$

Then,

$$
(\vartheta-1) E \int_{0}^{T} e^{-\vartheta t}|\widehat{X}(t)|^{2} d t \leq 6 C_{2} \cdot E \int_{-\delta}^{T} e^{-\vartheta t}|\widehat{\chi}(t)|^{2} d t .
$$

Let $\vartheta=12 C_{2}+1$, then the above yields

$$
E \int_{-\delta}^{T} e^{-9 t}|\widehat{X}(t)|^{2} d t \leq \frac{1}{2} E \int_{-\delta}^{T} e^{-9 t}|\widehat{\chi}(t)|^{2} d t
$$

That is,

$$
|\widehat{X}(\cdot)|_{\vartheta}=\frac{1}{\sqrt{2}}|\widehat{\chi}(\cdot)|_{\vartheta} .
$$

This implies that $\mathbb{T}$ is a strict contraction mapping. Then it follows from the fixed point theorem that (1)-(2) has a unique solution in $L_{\mathscr{F}}^{2}\left([-\delta, T] ; \mathbf{R}^{n}\right)$. Since $b$ and $\sigma$ satisfy $(\mathrm{H} 2.1)$ and (H2.2), we can easily derive that $E\left[|X(t ; \xi)|^{2}\right]<\infty$, and $x(t ; \xi)$ is continuous with respect to $t \in[0, T]$. Furthermore, by $b(t, 0,0,0)=\sigma(t, 0,0,0) \equiv 0$, (1)-(2) have a trivial solution $X(t ; 0)=0$. 
Proof of Lemma 6. For any $t \geq \delta$, we easily get

$$
\begin{aligned}
& \int_{\delta}^{t} e^{\varepsilon s} \int_{s-\delta}^{s} E|X(\tau)|^{2} d \tau d s \\
&= \int_{0}^{t} E|X(\tau)|^{2}\left(\int_{\tau \vee \delta}^{(\tau+\delta) \wedge t} e^{\varepsilon s} d s\right) d \tau \\
& \leq \delta e^{\varepsilon \delta} \int_{0}^{t} e^{\varepsilon \tau} \cdot E|X(\tau)|^{2} d \tau . \\
& \int_{0}^{t} e^{\varepsilon s} E|X(s-\delta)|^{2} d s \\
& \leq e^{\varepsilon \delta} \int_{0}^{\delta} E|X(s-\delta)|^{2} d s \\
& \quad+e^{\varepsilon \delta} \int_{\delta}^{t} e^{\varepsilon(s-\delta)} E|X(s-\delta)|^{2} d s \underline{\underline{\tau}=s-\delta} e^{\varepsilon \delta} \\
& \quad \times \int_{-\delta}^{0} E|\xi(\tau)|^{2} d \tau \\
& \quad+e^{\varepsilon \delta} \int_{0}^{t-\delta} e^{\varepsilon \tau} E|X(\tau)|^{2} d \tau \\
& \leq c_{11} e^{\varepsilon \delta}+e^{\varepsilon \delta} \int_{0}^{t} e^{\varepsilon \tau} E|X(\tau)|^{2} d \tau
\end{aligned}
$$

for any $t \geq \delta$, where $c_{11} \geq \int_{-\delta}^{0} E|\xi(\tau)|^{2} d \tau$.

Proof of Lemma 7. Similar to (11), for any $t \geq \delta$, we have

$$
\begin{aligned}
& \int_{\delta}^{t} e^{\varepsilon s} \int_{s-\delta}^{s} E|X(\tau-\delta)|^{2} d \tau d s \\
& \quad \leq \delta e^{\varepsilon \delta} \int_{0}^{t} e^{\varepsilon \tau} E|X(\tau-\delta)|^{2} d \tau .
\end{aligned}
$$

Substituting (12) into the above inequality yields

$$
\begin{aligned}
& \int_{\delta}^{t} e^{\varepsilon s} \int_{s-\delta}^{s} E|X(\tau-\delta)|^{2} d \tau d s \\
& \quad<\delta c_{11} e^{2 \varepsilon \delta}+\delta e^{2 \varepsilon \delta} \int_{0}^{t} e^{\varepsilon s} E|X(s)|^{2} d s .
\end{aligned}
$$

The relation (13) in Lemma 7 is then proved.

On the other hand, for $s \geq \delta$, we have

$$
\begin{aligned}
X(s)-X(s-\delta)= & \int_{s-\delta}^{s} b(t, X, Y) d t \\
& +\int_{s-\delta}^{s} \sigma(t, X, Y) d B(t) \\
& +\int_{s-\delta}^{s} \int_{\mathbf{R}^{n}} \gamma(t, X, Y, z) \widetilde{N}(d t, d z) .
\end{aligned}
$$

By (H2.1), we get

$$
\begin{aligned}
E|X-Y|^{2} \leq & 3 \delta E \int_{s-\delta}^{s}|b(t, X, Y)|^{2} d t \\
& +3 E \int_{s-\delta}^{s} \operatorname{tr}\left[\sigma^{T}(t, X, Y) \sigma(t, X, Y)\right] d t \\
& +3 E \int_{s-\delta}^{s} \int_{\mathbf{R}_{0}} \sum_{k=1}^{l}\left|\gamma^{(k)}\left(t, X, Y, z_{k}\right)\right|^{2} v_{k}\left(d z_{k}\right) d t \\
= & 3 \delta C_{1}(\delta+2)+3 C_{1}(\delta+2) \\
& \times \int_{s-\delta}^{s}\left(E|X|^{2}+E|Y|^{2}\right) d t .
\end{aligned}
$$

Similar to (12), for $t \geq \delta$, we have

$$
\begin{aligned}
\int_{0}^{t} e^{\varepsilon s} E|X-Y|^{2} d s & \\
= & \int_{0}^{\delta} e^{\varepsilon s} E|X(s)-X(s-\delta)|^{2} d s \\
& \quad+\int_{\delta}^{t} e^{\varepsilon s} E|X(s)-X(s-\delta)|^{2} d s \\
\leq & c_{22}+3 C_{1}(\delta+2) \int_{\delta}^{t} e^{\varepsilon s} \int_{s-\delta}^{s}\left(E|X|^{2}+E|Y|^{2}\right) d t d s
\end{aligned}
$$

where

$$
\begin{aligned}
c_{22} \geq & \int_{0}^{\delta} e^{\varepsilon s} E|X(s)-X(s-\delta)|^{2} d s \\
& +\frac{3 \delta C_{1}(\delta+2)}{\varepsilon}\left(e^{\varepsilon T}-e^{\varepsilon \delta}\right) .
\end{aligned}
$$

Substituting (11) and (13) into (A.16), for $t \geq \delta$, we get

$$
\begin{aligned}
\int_{0}^{t} e^{\varepsilon s} E|X(s)-X(s-\delta)|^{2} d s & \\
\leq & c_{22}+3 C_{1}(\delta+2) \delta e^{\varepsilon \delta} \int_{0}^{t} e^{\varepsilon s} E|X(s)|^{2} d s \\
& +3 C_{1}(\delta+2)\left(c_{11} \delta e^{2 \varepsilon \delta}+\delta e^{2 \varepsilon \delta} \int_{0}^{t} e^{\varepsilon s} E|X(s)|^{2} d s\right) .
\end{aligned}
$$

\section{Conflict of Interests}

The authors declare that there is no conflict of interests regarding the publication of this paper.

\section{Acknowledgment}

This work is supported by the Fundamental Research Funds for the Central Universities (JBK140205). 


\section{References}

[1] K. Itô, "Differential equations determining Markov processes," Zenkoku Shijo Sugaku Danwakai, vol. 244, no. 1077, pp. 1352$1400,1942$.

[2] S. Cong, "On exponential stability conditions of linear neutral stochastic differential systems with time-varying delay," International Journal of Robust and Nonlinear Control, vol. 23, no. 11, pp. 1265-1276, 2013.

[3] Q. Zhu and X. Li, "Exponential and almost sure exponential stability of stochastic fuzzy delayed Cohen-Grossberg neural networks," Fuzzy Sets and Systems, vol. 203, pp. 74-94, 2012.

[4] Y. Sun and J. Cao, "pth moment exponential stability of stochastic recurrent neural networks with time-varying delays," Nonlinear Analysis: Real World Applications, vol. 8, no. 4, pp. 1171-1185, 2007.

[5] Q. Zhu and B. Song, "Exponential stability of impulsive nonlinear stochastic differential equations with mixed delays," Nonlinear Analysis: Real World Applications, vol. 12, no. 5, pp. 2851-2860, 2011.

[6] X. Mao and A. Shah, "Exponential stability of stochastic differential delay equations," Stochastics and Stochastics Reports, vol. 60, no. 1-2, pp. 135-153, 1997.

[7] W. Zhu, X. Ruan, Y. Qin, and J. Zhuang, "Exponential stability of stochastic nonlinear dynamical price system with delay," Mathematical Problems in Engineering, vol. 2013, Article ID 168169, 9 pages, 2013.

[8] W. Zhu and J. Hu, "Stability analysis of stochastic delayed cellular neural networks by LMI approach," Chaos, Solitons and Fractals, vol. 29, no. 1, pp. 171-174, 2006.

[9] B. Øksendal and A. Sulem, "A maximum principle for optimal control of stochastic systems with delay, with applications to finance," in Optimal Control and Partial Differential Equations, J. M. Menaldi, E. Rofman, and A. Sulem, Eds., pp. 64-79, ISO Press, Amsterdam, The Netherlands, 2000.

[10] B. Øksendal and A. Sulem, Applied Stochastic Control of Jump Diffusions, Universitext, Springer, Berlin, Germany, 2nd edition, 2007.

[11] F. E. Benth and T. Meyer-Brandis, "The density process of the minimal entropy martingale measure in a stochastic volatility model with jumps," Finance and Stochastics, vol. 9, no. 4, pp. 563-575, 2005.

[12] D. Bates, "The crash of '87: Was it expected? The evidence from options markets," Journal of Finance, vol. 46, no. 3, pp. 10091044, 1991.

[13] L. Guo and Q. Zhu, "Stability analysis for stochastic VolterraLevin equations with Poisson jumps: fixed point approach," Journal of Mathematical Physics, vol. 52, no. 4, Article ID 042702, 2011.

[14] Q. Zhu and J. Cao, "Robust exponential stability of markovian jump impulsive stochastic Cohen-Grossberg neural networks with mixed time delays," IEEE Transactions on Neural Networks, vol. 21, no. 8, pp. 1314-1325, 2010.

[15] Q. Zhu and J. Cao, "Exponential stability of stochastic neural networks with both Markovian jump parameters and mixed time delays," IEEE Transactions on Systems, Man, and Cybernetics B: Cybernetics, vol. 41, no. 2, pp. 341-353, 2011.

[16] Q. Zhu, "Asymptotic stability in the pth moment for stochastic differential equations with Lévy noise," Journal of Mathematical Analysis and Applications, vol. 416, no. 1, pp. 126-142, 2014.

[17] L. Chen, M. Chen, and L. Guo, "Exponential stability of impulsive stochastic delay partial differential equations with
Poisson jumps," Journal of Ningbo University, vol. 25, no. 3, pp. 30-34, 2012.

[18] S. Boyd, L. El Ghaoui, E. Feron, and V. Balakrishnan, Linear Matrix Inequalities in System and Control Theory, vol. 15 of SIAM Studies in Applied Mathematics, SIAM, Philadelphia, Pa, USA, 1994.

[19] V. B. Kolmanovskii and V. R. Nosov, Stability of Functional Differential Equations, Academic Press, 1986.

[20] Y. Hu and F. Wu, "Stochastic Kolmogorov-type population dynamics with infinite distributed delays," Acta Applicandae Mathematica, vol. 110, no. 3, pp. 1407-1428, 2010. 


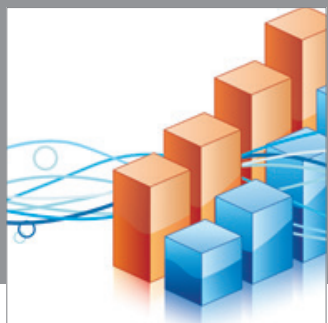

Advances in

Operations Research

mansans

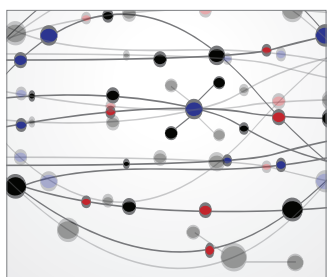

The Scientific World Journal
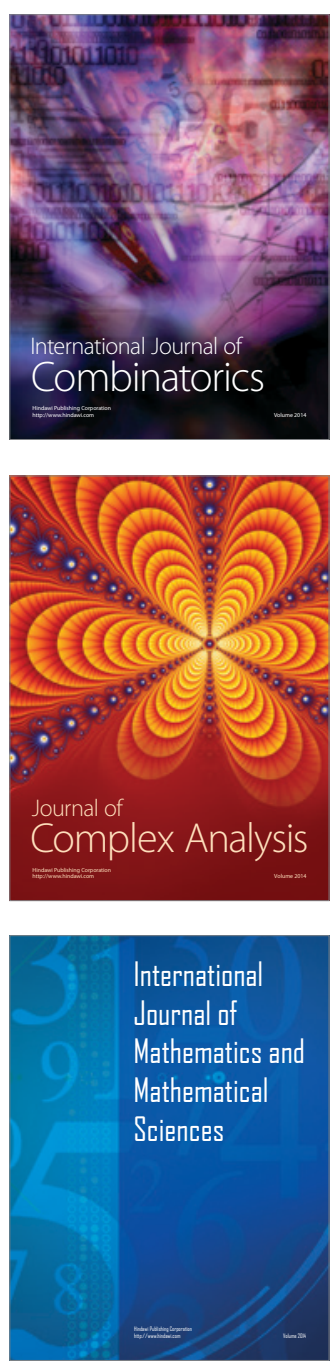
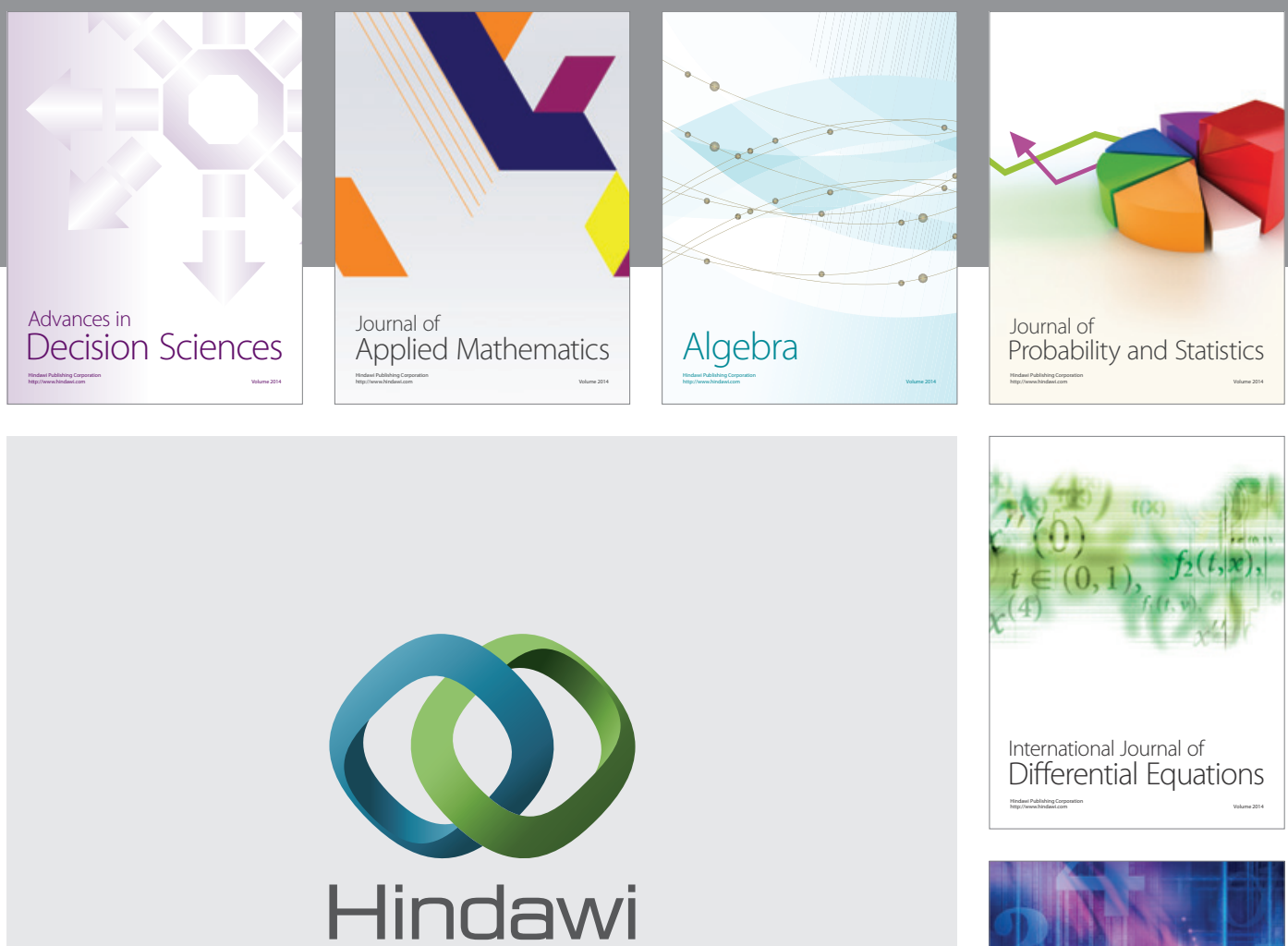

Submit your manuscripts at http://www.hindawi.com
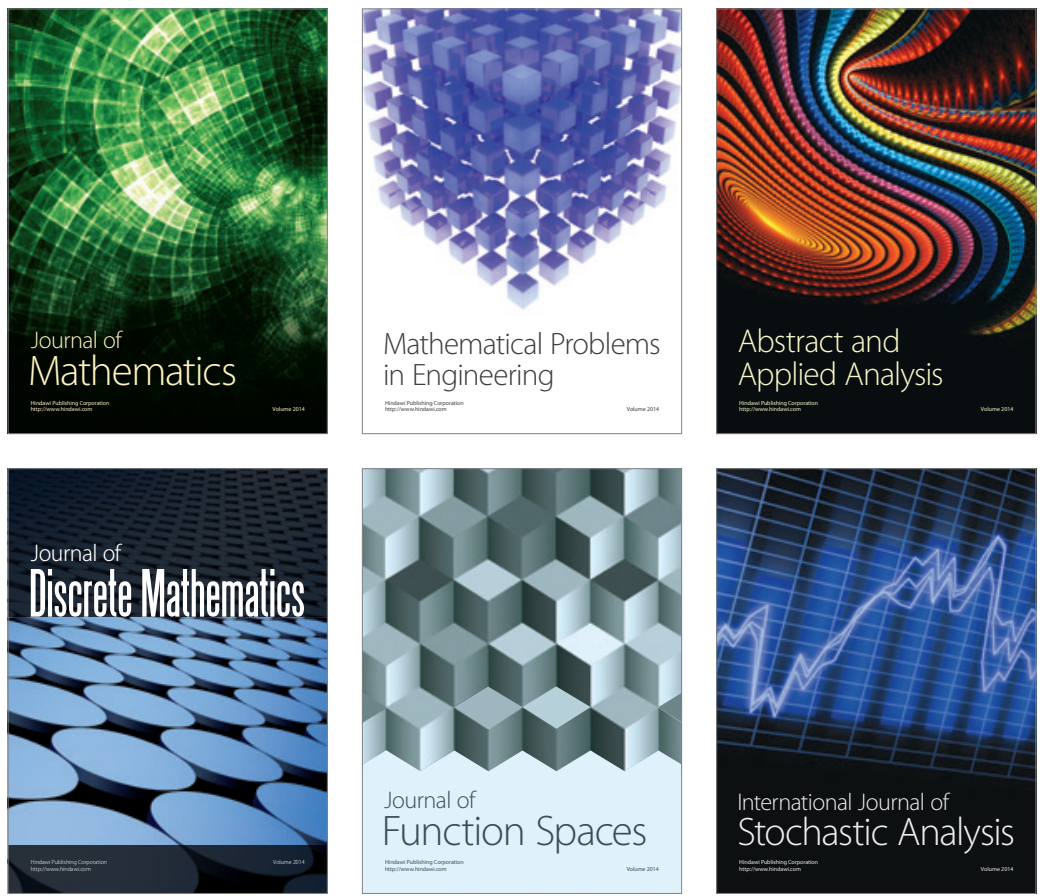

Journal of

Function Spaces

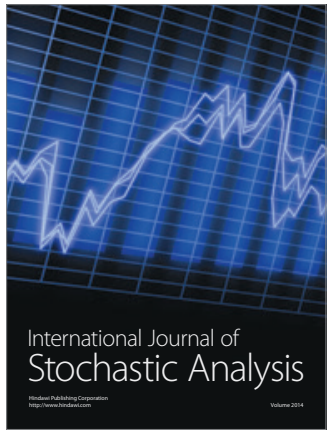

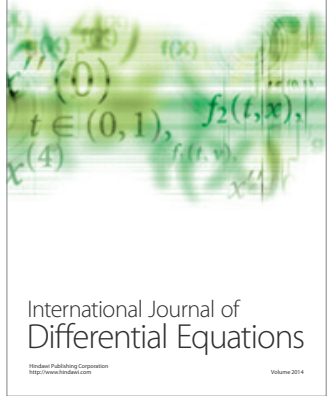
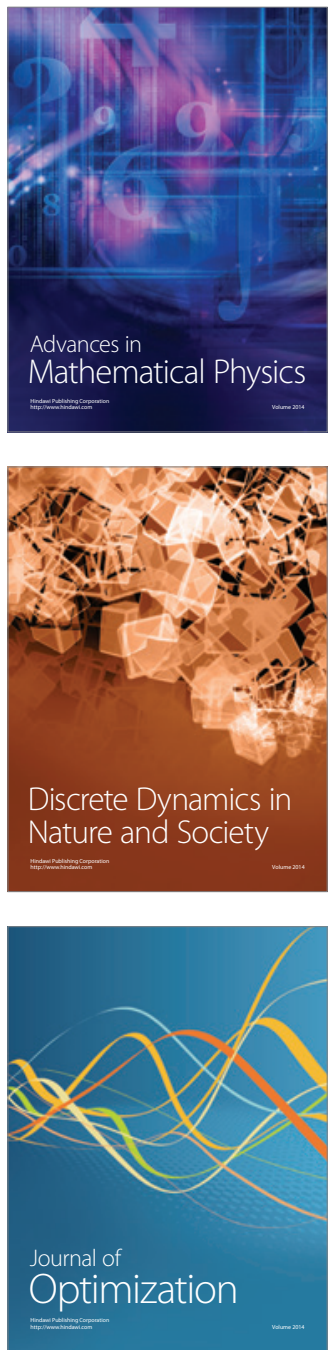